\title{
Formation of brainstem (nor)adrenergic centers and first-order relay visceral sensory neurons is dependent on homeodomain protein Rnx/Tlx3
}

\author{
Ying Qian, ${ }^{1,2}$ Bernd Fritzsch, ${ }^{3}$ Senji Shirasawa, ${ }^{4}$ Chih-Li Chen, ${ }^{1,2}$ Yoojin Choi, ${ }^{2}$ and Qiufu $\mathrm{Ma}^{1,2,5}$ \\ ${ }^{1}$ The Dana-Farber Cancer Institute and ${ }^{2}$ Department of the Neurobiology, Harvard Medical School, Boston, Massachusetts \\ 02115, USA; ${ }^{3}$ Department of Biomedical Sciences, Creighton University, Omaha, Nebraska 68178, USA; ${ }^{4}$ Department of \\ Pathology, International Medical Center of Japan, Tokyo 162-8655, Japan
}

Brainstem visceral sensory and (nor)adrenergic neurons play crucial roles in modulating cardiovascular and respiratory functions. The origins and formation of these neurons are poorly understood. Here we show that these two classes of neurons are derived from Mash1-positive precursor cells, and can be prospectively identified by combinatorial expression of two homeobox genes, Rnx and Phox2 (Phox2a or Phox2b). It was previously shown that $R n x$-deficient mice die from respiratory failure. Here we show that Rnx function is required for formation of first-order relay visceral sensory neurons in the brainstem. In addition, as in Phox $2 b$-deficient mice, the development of most (nor)adrenergic centers is compromised in Rnx mutants. We also provide genetic evidence to show that Rnx and Phox 2 proteins may function independently to specify the (nor)adrenergic phenotype. Our studies reveal a surprising ontogenetic relationship between relay visceral sensory and (nor)adrenergic neurons, and suggest that it may be a common theme in the developing nervous system that the same set of transcriptional regulators is associated with formation of multiple components within a neuronal network.

[Key Words: Noradrenergic; sensory neurons; respiratory control; Rnx; Phox2b; Mash1]

Received June 20, 2001; revised version accepted August 17, 2001.

Physiological control of cardiovascular and respiratory systems involves complex neuronal circuits. Respiration patterns are generated by rhythmic neurons in the ventral medulla and modulated by input from other groups of neurons in the brainstem, including visceral sensory and (nor)adrenergic neurons (i.e., adrenergic and noradrenergic neurons) (Fig. 1; Blessing 1997; Rekling and Feldman 1998). For instance, activation of peripheral chemoreceptors and pulmonary stretch receptors, carried via first-order relay visceral sensory neurons in the nucleus of the solitary tract (nTS), leads to an increase of respiration rate (Fig. 1; Blessing 1997). In contrast, input from (nor)adrenergic neurons predominantly causes a depression of respiratory frequency (Fig. 1; Bolme and Fuxe 1973; Champagnat et al. 1979; Errchidi et al. 1991; AlZubaidy et al. 1996).

Despite the pivotal roles played by visceral sensory and (nor)adrenergic neurons in modulating cardiorespiratory activities, the development of these two classes of

${ }^{5}$ Corresponding author.

E-MAIL Qiufu_Ma@dfci.harvard.edu; FAX (617) 632-4595.

Article and publication are at http://www.genesdev.org/cgi/doi/10.1101/ gad.921501. neurons is poorly understood. At the molecular level, the most important progress made so far is the identification and characterization of two closely related genes Phox $2 a$ and Phox $2 b$, which encode a paired-like class of homeodomain proteins (Valarché et al. 1993; Yokoyama et al. 1996; Pattyn et al. 1997). Phox2 proteins are required for formation of the (nor)adrenergic neurons throughout the nervous system (Morin et al. 1997; Pattyn et al. 1999, 2000a). However, Phox2 genes are also associated with non-(nor)adrenergic structures, including hindbrain parasympathetic preganglion motor neurons and branchial motor neurons (Valarché et al. 1993; Tiveron et al. 1996; Pattyn et al. 1997, 2000b), suggesting that they are necessary but not sufficient to specify the (nor)adrenergic phenotype. Consistent with this, ectopic expression of Phox $2 a$ in Zebrafish embryos induces (nor)adrenergic neurons only in highly restricted locations /Guo et al. 1999). Neural crest cell culture studies also show that Phox 2 a requires additional signals to induce the (nor)adrenergic feature (Lo et al. 1999).

Thus far, the factors that work cooperatively with Phox 2 proteins in the specification of (nor)adrenergic neurons remain to be identified. The proneural protein MASH1 has been shown to be required for the formation 


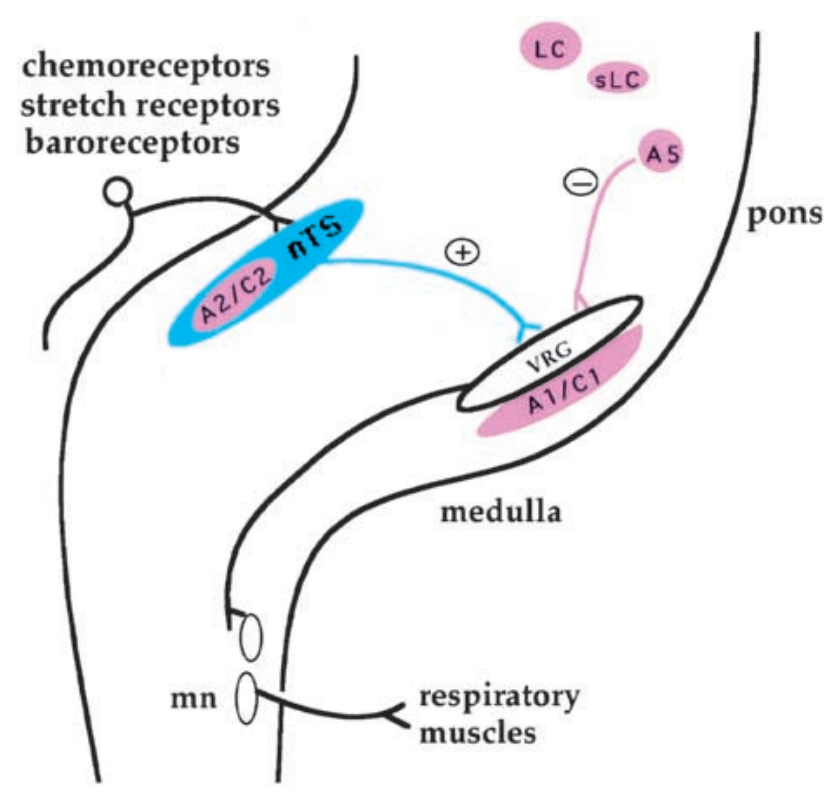

Figure 1. Schematic of respiratory neuronal circuitry. The basic breathing rhythm is generated by respiratory neurons located in the ventral medulla (VRG). The activation of peripheral chemoreceptors by a high carbon dioxide level in the blood flow, that of stretch receptors by air inflation in the lung, or that of baroreceptors by high blood pressure, provides stimulation of respiration rates. The peripheral stimulatory signals are carried via first-order relay visceral sensory neurons in the nucleus of the solitary tract (nTS). The nTS, in turn, makes connections to the VRG. Via further relays through spinal respiratory motor neurons $(\mathrm{mn})$, the activities of respiratory muscles will be modulated. In contrast, inputs from (nor)adrenergic centers (in pink) cause a depression of respiration frequency. (Nor)adrenergic neurons are divided into two classes, namely adrenergic $(\mathrm{C} 1$ and $\mathrm{C} 2$ ) and noradrenergic (A1, A2, A5, sLC, and LC). sLC, Locus subcoeruleus; LC, locus coeruleus.

of most (nor)adrenergic neurons (Hirsch et al. 1998), but MASH1 function is primarily mediated by regulating the expression of Phox2 genes (Hirsch et al. 1998; Pattyn et al. 2000a). In vitro promoter analyses show that the retinoic acid inducible transcription factor, AP2, may be involved in regulation of the (nor)adrenergic phenotype (Kim et al. 2001), but its in vivo requirement is unknown.

The molecular basis of the formation of relay visceral sensory neurons is even less understood. Phox $2 b$ is expressed in the nTS (Pattyn et al. 1997), but its function in these neurons has not been characterized. Tlx is another family of homeobox genes expressed in restricted regions of the developing hindbrain. There are three family members, Hox11/Tlx1, Hox11-L1/Tlx2/Enx, and Hox11L2/T1x3/Rnx (Dube et al. 1991; Hatano et al. 1991, 1997; Kennedy et al. 1991; Dear et al. 1993, 1995; Raju et al. 1993; Roberts et al. 1994, 1995; Shirasawa et al. 1997, 2000; Logan et al. 1998; Tang et al. 1998). For simplicity, these three genes will be referred to here as Tlx1, Enx, and $R n x$, respectively. In both chick and mouse embryos, $R n x$ is expressed in two longitudinal stripes of postmitotic neurons in the developing hindbrain and spinal cord (Logan et al. 1998; Shirasawa et al. 2000). It was hypothesized that part of the ventral stripe may give rise to the nTS in chick embryos (Logan et al. 1998). However, because of extensive cell migration and complex morphogenesis in the developing hindbrain (Altman and Bayer 1987), it has been difficult to definitely assign cell fates if prospective markers for the cells of interest have not been established.

$R n x$-deficient mice show a phenotype resembling the congenital hypoventilation syndrome in humans, and all die within $24 \mathrm{~h}$ after birth from respiratory failure (Shirasawa et al. 2000). Here we show that $R n x$ is expressed in, and required for proper formation of, first-order relay visceral sensory neurons and most of the (nor)adrenergic centers in the brainstem. All of these neurons can be prospectively defined by a combinatorial expression of $R n x$ and Phox $2 b / P h o x 2 a$ at a time when they are formed. Furthermore, we show that $R n x$ and Phox 2 genes are required independently for specification of the (nor)adrenergic phenotype. These findings establish a central role of $R n x$ in the formation of two major classes of modulator neurons in the brainstem that are crucial for cardiorespiratory control.

\section{Results}

\section{Coexpression of $\mathrm{Rnx}$ and Phox $2 \mathrm{~b}$ in developing nTS}

$R n x$-deficient mice die from a hypothesized central defect in respiratory control (Shirasawa et al. 2000). Since the Phox2 genes are expressed in and required for proper development of many visceral sensory and autonomic neurons (Morin et al. 1997; Pattyn et al. 1999, 2000a), we performed a detailed examination of $R n x$ expression in relation to that of Phox $2 a$ and Phox $2 b$. As in chick embryos (Logan et al. 1998), whole mount in situ hybridization of embryonic day (E)9.5-E11 mouse embryos reveals that $R n x$ is initially expressed in two longitudinal stripes (Fig. 2A,B; data not shown). This can be seen best in neurogenin1 mutants due to the absence of a subset of peripheral ganglia (Fig. 2B, arrows and arrowheads; Ma et al. 1998). The dorsal stripe of $R n x$ expression is first detected in E10.5 mouse embryos (data not shown), and by E11, it appears to overlap that of Phox2b (Fig. 2B,C, arrowheads; also see below). The rostral extent of $P$ hox $2 b$ and $R n x$ expression is at the axial level where the geniculate ganglion is located (Fig. 2B,C, g). Caudally, Phox2b expression ends around the obex, the junction between the spinal cord and the hindbrain. Thus, the anterior posterior extent of Phox $2 b$ expression in the dorsal hindbrain appears to correspond to the location of the nTS (Saper 2000).

By E11.5, neurons derived from the Rnx/Phox $2 b$ dorsal stripe appear to migrate in a ventral direction, as shown by in situ hybridization on adjacent transverse sections (Fig. 2D,E, black arrows). Coexpression of $R n x$ and $P h o x 2 b$ in these cells is demonstrated by double labeling, with $R n x$ mRNA detected by in situ hybridization and Phox $2 \mathrm{~b}$ protein detected by immunostaining (Fig. $2 \mathrm{~F}, \mathrm{G})$. In contrast, the ventral stripe of $R n x$-expressing 

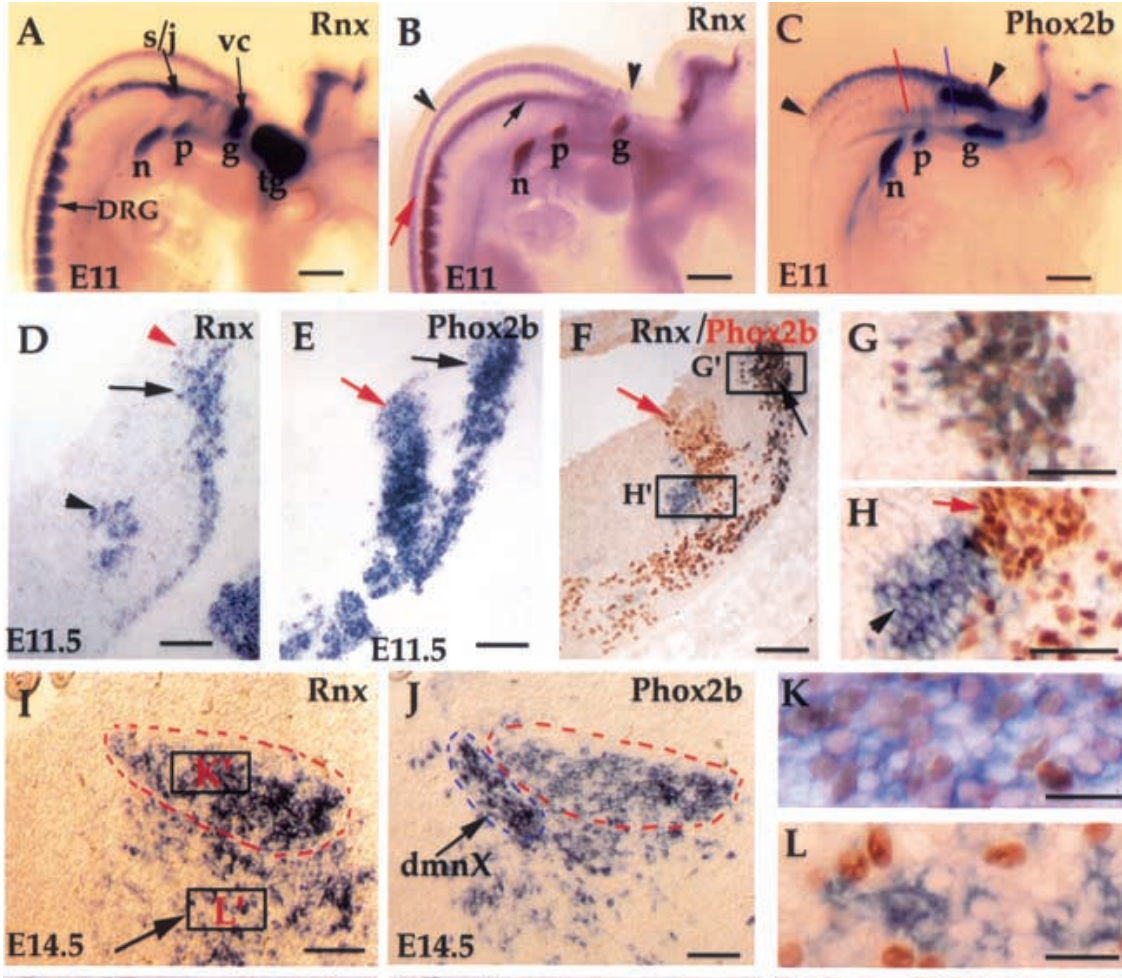

E11
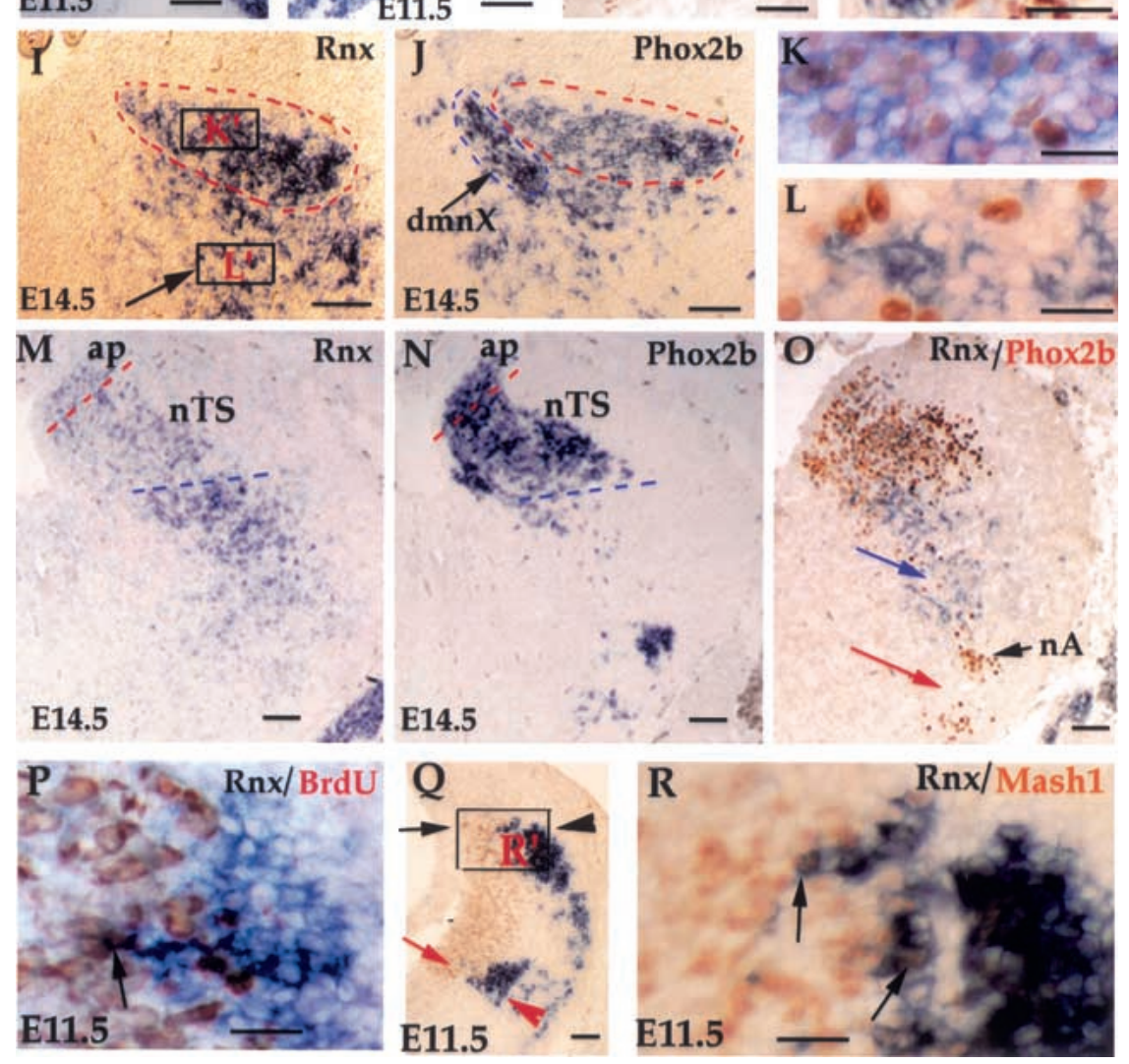

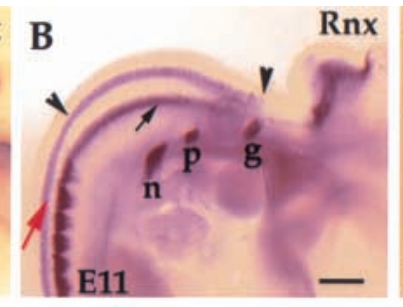

Figure 2. Coexpression of $R n x$ and Phox $2 b$ in, and developmental origin of, the nTS. $(A-C)$ Whole mount in situ hybridization with indicated probes. E11 wild-type $(A, C)$ and ngn1 mutant $(B)$ embryos. $R n x$ is expressed in every sensory ganglion $(A)$, including trigeminal $(\mathrm{tg})$, geniculate $(\mathrm{g})$, pertrosal $(\mathrm{p})$, nodose $(\mathrm{n})$, vestibular-cochlear (vc), and superior/jugular ganglia (s/j). Rnx expression in the central nervous system is seen best in ngn1 null mutants because of the absence of sensory ganglia (cf. $A$ and $B$ ). The dorsal expression of Rnx and Phox $2 b$ seems to overlap $(B, C$, arrowheads) (see below). The fates of the ventral stripe of $R n x$-expressing cells in the brainstem $(B$, arrow) as well as the longitudinal stripes in the spinal cord $(B$, red arrow) will be reported elsewhere. The blue and red lines in panel $C$ represent the axial levels for the sections shown in panels $D-L$ and $M-O$, respectively. $(D-O)$ Adjacent transverse sections through E11.5 embryos $(D-L)$ and E14.5 embryos $(I-O)$. Dorsal is up and lateral is to the right. In situ hybridization was performed with indicated probes. Double labeling was performed on sections $F-H, K, L$, and $O$, with $R n x$ mRNA detected by in situ hybridization (purple staining in the cytoplasm), and Phox $2 \mathrm{~b}$ protein detected by immunostaining with Phox $2 b$ antibody (brown staining in the nucleus). Expression of $R n x$ and Phox $2 b$ in the dorsal position $(D, E$, black arrows) overlaps, as demonstrated by double labeling $(F, G)$. (Panel $G$ corresponds to box $G^{\prime}$ shown in panel $F$ ). In contrast, the ventral $R n x$ stripe $(D$, arrowhead) and the intermediate Phox $2 b$ stripe $(E$, red arrow) do not overlap $(F, H)$. (Panel $H$ is a higher magnification of box $H^{\prime}$ shown in panel F). By E14.5, neurons coexpressing $R n x$ and Phox $2 b$ are located in a region that corresponds to the nTS $(I, I$, dashed red circles), which is demonstrated by double staining $(K)$. The nTS is distinct from the adjacent location of the dorsal motor nucleus of the vagus $(H, \mathrm{dmnX})$, which itself is labeled by in situ hybridization on an adjacent section with choline acetyl transferase as the probe (Tiveron et al., 1996; data not shown). Again in the more ventral area ( $I$, arrow), the expression of $R n x$ and Phox $2 b$ does not overlap $(L)$. (Panel $L$ corresponds to box $L^{\prime}$ in panel $\left.I\right)$. Rnx expression is reduced significantly in caudal nTS $(M, \mathrm{nTS})$, starting at the axial level of area postrema $(M$, ap). Weak Rnx expression is also detected in the ap $(M$, ap). In contrast, strong Phox2b expression is detected at any axial level examined $(J, N)$. Panel $O$ shows double staining of $R n x$ and Phox $2 b$. The most ventral $R n x$ expression $(O, b l u e$ arrow $)$ is located in the area dorsal to the nucleus ambiguus (nA), which is a dense cluster of Phox 2 b-expressing branchial motor neurons $(O$, nA) (Pattyn et al. 2000b). No $R n x$ expression is detected in the area ventral to the $n A(O$, red arrow). $(P-R)$ Transverse sections through the developing medulla of E11.5 wild-type embryos. Panel $P$ shows $R n x$ in situ hybridization (purple) followed by anti-BrdU immunostaining (brown) (the section roughly corresponds to the boxed region shown in panel $Q)$. No double staining is detected $(P)$, except for a single cell ( $P$, arrow). Panels $Q$ and $R$ show double staining of $R n x$ mRNA (purple) and MASH1 protein (brown). Panel $R$ is a high magnification of the boxed region shown in panel $Q$. MASH1 is expressed in precursor cells located in the ventricular zone $/ Q, R$, brown staining). The dorsal ( $Q$, black arrowhead) and ventral ( $Q$, red arrowhead) stripes of $R n x$-expressing cells are located lateral to the most dorsal $(Q$, black arrow $)$ and the most ventral $(Q$, red arrow) parts of the MASH1-expressing domain, respectively. A few double-stained cells were detected $(R$, arrows). Bars: $A-C, 200 \mu \mathrm{m} ; K, L, P, R, 50 \mu \mathrm{m}$; all remaining panels, $100 \mu \mathrm{m}$. 
cells (Fig. 2D, arrowhead) and the intermediate stripe of Phox2b-expressing cells (Fig. 2E, red arrow) do not overlap (Fig. 2F,H).

By E14.5, cells coexpressing Rnx and Phox $2 b$ are located in a region corresponding to the nTS (Fig. 2I-O). At this stage, Rnx expression in the caudal part of the nTS is significantly reduced (Fig. 2, cf. M and I, nTS). Weak $R n x$ expression is also detected in the area postrema (Fig. $2 \mathrm{M}$, ap). (The area postrema, which is located dorsalmedial to the nTS, is innervated by a subset of primary visceral sensory afferents important for cardiovascular control; Blessing 1997). In contrast, strong Phox $2 b$ expression is detected throughout the entire nTS (Fig. 2J,N). In summary, the dorsal stripe of $R n x$-expressing cells seen in E11 mouse embryos (Fig. 2B, arrowheads) is fated to form the relay visceral sensory neurons in the nTS, and possibly the area postrema, rather than the nearby vestibular nucleus proposed in chick embryos (Logan et al. 1998).

It has been hypothesized that the breathing problem in $R n x$-deficient mice is caused by a functional disorder of ventral respiratory neurons (Shirasawa et al. 2000). The rhythmic respiratory neurons are normally located in a region ventral to the nucleus ambiguus (nA) (Rekling and Feldman 1998). The $\mathrm{nA}$ is a dense cluster of branchial motor neurons that can be recognized by the expression of Phox2b (Fig. 2O, nA) (Pattyn et al. 2000b). We find that persistent $R n x$ expression is not detected in the area ventral to the nA (Fig. 2O, red arrow). This result suggests that $R n x$ is either not expressed, or expressed transiently in ventral respiratory neurons.

\section{The nTS develops from Mash1-expressing neural precursors}

We note that in E11.5 mouse embryos, a few Rnx-expressing cells are located within the ventricular zone (Fig. 2D, red arrowhead). Double labeling, with $R n x$ mRNA detected by in situ hybridization and dividing cells detected by anti-BrdU immunostaining, shows that $R n x$ is predominantly expressed in postmitotic cells (Fig. 2P). However, occasionally we do observe double-labeled cells (Fig. 2P, arrow), suggesting that $R n x$ expression is initiated right after cells exit from the cell cycle. Thus, the nTS neurons are likely derived from the ventricular zone medial to the most dorsal Rnx-expressing cells.

The ventricular zone in the developing neural tube is subdivided to distinct territories that express, from dorsal to ventral, Math1, ngn1, and Mash1, respectively (Gradwohl et al. 1996; Ma et al. 1997; Gowan et al. 2001). We find that the dorsal stripe of $R n x$-expressing cells is located lateral to the MASH1-expressing territory (Fig. 2J, black arrow). Moreover, coexpression of MASH1 protein and Rnx mRNA is detected in a few cells within the subventricular zone (Fig. $2 \mathrm{~K}$, arrows). We conclude that dorsally derived $R n x$-expressing cells, which are fated to form the nTS, develop from the most dorsal MASH1positive territory. Interestingly, the ventral stripe of $R n x$-expressing cells apparently develops from the most ventral part of the MASH1-positive domain (Fig. 2Q, red arrow).

\section{Development of the nTS is compromised in $\mathrm{Rnx}$ mutant mice}

The nTS is a key component of the respiratory neuronal circuitry (Fig. 1). Indeed, we find that development of the nTS is compromised in Rnx-deficient mice. In E19 Rnx mutant mice, Phox $2 b$ expression in the prospective nTS area is nearly completely eliminated (Fig. 3A-F). This is true at all axial levels examined (Fig. 3A-F). In addition, Phox $2 b$ expression in the area postrema is also missing (Fig. 3, cf. C and D, ap). In contrast, expression of Phox $2 b$ in other brainstem structures is not affected, including

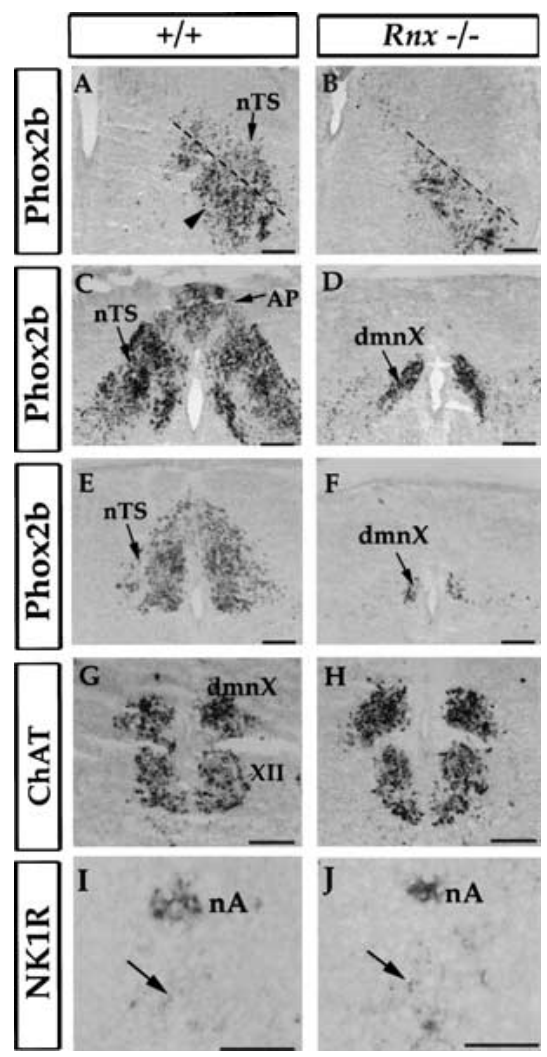

Figure 3. Loss of Phox $2 b$ expression in the nTS of E19 Rnx mutant embryos. Adjacent transverse sections through E19 wild-type and mutant embryos. In situ hybridization was performed with indicated probes. Phox $2 b$ expression is lost throughout the entire nTS $(A-F, \mathrm{nTS})$, including the rostral part of the nTS $(A, B)$, medial part of the nTS (at the axial level of area postrema) $(C, D)$, and caudal commissural nTS $(E, F)$. No defects were detected in other Phox $2 b$-expressing structures, including a group of neurons located ventral to the rostral nTS $(A$, arrowhead), and the dorsal motor nucleus of the vagus $(C-F, \mathrm{dmnX})$. The normal formation of the $\mathrm{dmnX}$ and hypoglossal motor nucleus $(G, \mathrm{XII})$ is also indicated by expression of $C h A T$ ( $G$ and $H, \mathrm{dmnX})$. The expression of neurokinin-1 receptor (NK1R) in the nucleus ambiguus $(I, J, \mathrm{nA})$ and in the prospective respiratory pacemaker neurons $(I, J$, arrows) is not affected in RnX-deficient mice (cf. $J$ and $I$ ). Bars, $200 \mu \mathrm{m}$. 
the dorsal motor nucleus of vagus $(\mathrm{dmnX})$ (Fig. 3D,F, $\mathrm{dmnX}$ ) and a group of neurons located ventral to the rostral part of the nTS (Fig. 3A, arrowhead). The proper development of dmnX is further indicated by normal ChAT expression (Fig. 3G,H). Expression of neurokinin-1 receptor, a possible marker for ventral respiratory pacemaker neurons (Gray et al. 1999), is also not affected in Rnx-deficient mice (Fig. 3J,I, arrows).

To determine the developmental stages when the nTS defect occurs, we examined Phox $2 b$ expression in E11E16.5 Rnx mutant embryos. As described above, neurons in the nTS are formed in a dorsal position and then migrate ventrally (Fig. 2). In both E11 (data not shown) and E12.5 Rnx mutants, Phox $2 b$ expression in the most dorsal position (Fig. 4B, arrowhead) and in early migratory neurons (Fig. 4B, red arrow) is normal. However, in the area corresponding to the cells that have migrated to the more ventral position, Phox $2 b$ expression is eliminated (Fig. 4A,B, arrows). Consistently, BrdU pulse-chase labeling shows that by E12.5, Phox2b expression is no longer detected in the cells born at E10.5 (Fig. 4, cf. C and D, red arrows). Nonetheless, the detection of early Phox $2 b$ expression suggests that the nTS neurons are formed in

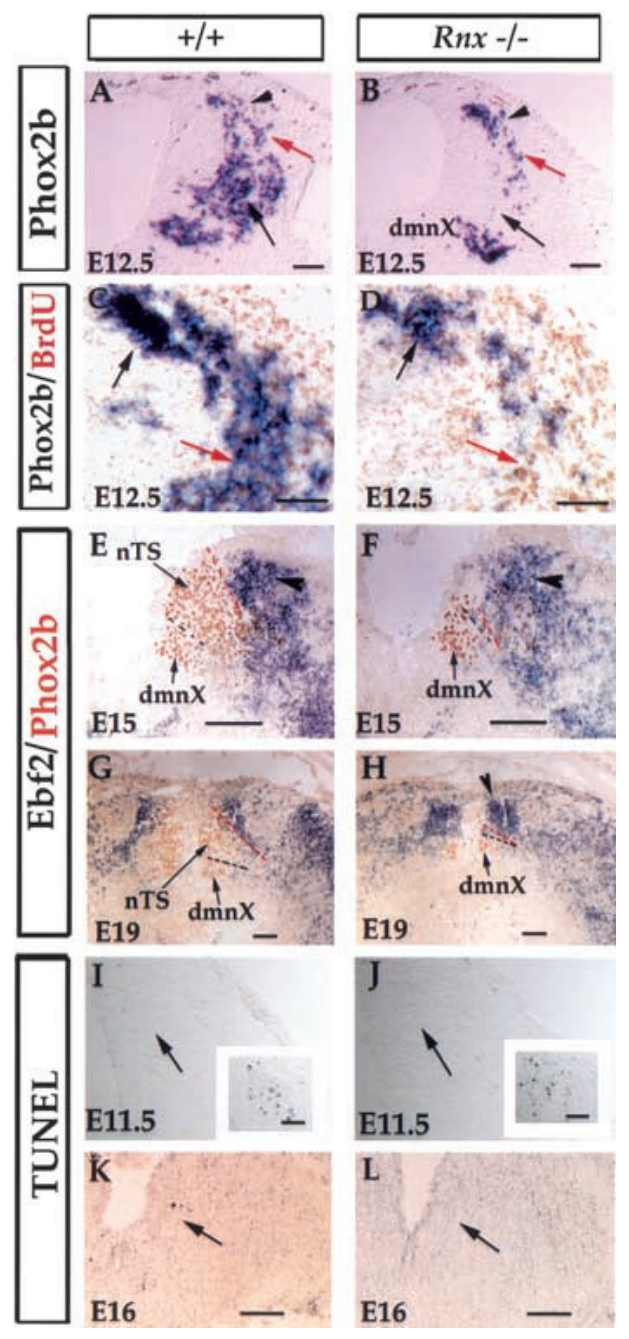

$R n x$ mutants, consistent with the finding that $R n x$ is predominantly expressed in postmitotic cells (Fig. 2).

The eventual loss of Phox $2 b$ expression in Rnx mutants could be explained by two scenarios: loss of mutant cells by cell death or a later role of Rnx protein in maintaining Phox $2 b$ expression. To help distinguish these two possibilities, we took an indirect approach by examining molecular markers expressed in regions surrounding the nTS. Ebf2, a basic helix-loop-helix gene (Garel et al. 1997), is expressed in the region dorsal lateral to the nTS (Fig. 4E, arrowhead), whereas the dorsal motor nucleus of vagus (dmnX), which expresses $P h o x 2 b$, is ventral to the nTS (Fig. 4E, dmnX). Strikingly, in both E15 and E19 Rnx mutant embryos, the dmnX approximates Ebf2-expressing cells (Fig. 4F,H), suggesting that the intervening nTS structure is absent. To determine whether the mutant neurons die by apoptosis, we performed TUNEL labeling experiments (Gavrieli et al. 1992). Surprisingly, increased cell death was not detected in the prospective mutant nTS area at any stage examined, from E10.5 to P0 (Fig. 4I-L; data not shown). Thus, mutant neurons in the prospective nTS may either die through a pathway not detected by TUNEL labeling, or

Figure 4. The nTS neurons are generated in Rnx mutants, but are apparently lost at later stages. $(A, B)$ In situ hybridization on transverse sections through developing medulla of E12.5 Rnx wild-type $(A)$ and mutant $(B)$ embryos. Initiation of $P$ hox $2 b$ expression in the most dorsal part of the developing hindbrain is not affected in Rnx mutants (cf. $A$ and $B$, arrowheads). Nor is its expression in early migratory neurons affected (cf. $A$ and $B$, red arrows). However, Phox $2 b$ expression is absent in a more ventral area (cf. $A$ and $B$, black arrows). $(C, D)$ Pulse-chase BrdU labeling in Phox $2 b$-positive neurons. Transverse sections through E12.5 wild-type $(C)$ and $R n x$ mutant $(D)$ embryos. BrdU was injected at E10.5 and embryos were collected at E12.5. In situ hybridization with Phox $2 b$ probe (purple) followed by antiBrdU immunostaining (brown). Phox2b-positive cells born at E10.5 are recognized by strong BrdU staining, and are located in the ventral position in wild-type embryos $(C$, red arrow), but are absent in $R n x$ mutants ( $D$, red arrow). In contrast, cells born after E10.5 are located in the dorsal position and show either no or weak BrdU staining $(C, D$, black arrows). $(E-H)$ The nTS structure may be missing in Rnx mutants. Double staining of Ebf2 mRNA (purple) and Phox $2 \mathrm{~b}$ protein (brown). Transverse sections through medulla of E15 and E19 wild-type $(E, G)$ and $R n x$ mutant $(F, G)$ embryos. Ebf2 expression (E, arrowhead) is located dorsal lateral to the nTS in wild-type embryos $(E, G$, redlines). The dmnX, as indicated by Phox $2 \mathrm{~b}$ expression in Rnx mutants $(F, H, \mathrm{dmnX})$, is located ventral to the nTS in wild-type embryos $(E, G, \mathrm{dmnX})$. In E15 and E19 mutants, the dmnX abuts Ebf2expressing cells $(F, H, d m n X)$. At later stages, Ebf2-expressing cells appear reorganized in $R n x$ mutants, as indicated by an ectopic aggregation in the dorsal medial region $(F$, arrowhead). $(I-L)$ TUNEL labeling was performed on transverse sections through E11.5 and E16 wild-type $(I, K)$ and $R n x$ mutants $(J, L)$. No increased cell death was detected in the prospective nTS areas of $R n x$ mutants (cf. $I, K$ and $J, L$, arrows). The detection of extensive cell death in the accessory ganglia $(I, J$, boxed regions) serves as the positive control. No differential cell death was detected in other developmental stages (data not shown). Bars: $A-D, I, J, 100$ $\mu \mathrm{m} ; \mathrm{E}-H, 400 \mu \mathrm{m} ; K, L, 200 \mu \mathrm{m}$. 
Qian et al.

be converted to other cell fates. Potential support for the latter scenario is that there is an ectopic aggregation of Ebf2-expressing cells in the dorsal medial region of the medulla (Fig. 4H, arrowhead), although this could be simply due to a relocation of Ebf2-expressing cells.

\section{Aberrant central innervation of primary visceral sensory afferents in Rnx mutants}

Given the dramatic absence or reorganization of the nTS structure, we asked whether central innervation of the primary visceral sensory neurons is affected in $R n x$ mutants. The innervation pattern was analyzed by DiI-labeling. In wild-type embryos, after entering the hindbrain, the visceral sensory afferents are bundled together to form the descending solitary tract $(\mathrm{ST})$, and then make collateral projections to the nTS. We found that formation of the ST is not affected in E12.5 Rnx mutants (Fig. $5 \mathrm{~A}, \mathrm{~B})$. At E16, the initial collateral projection from the $\mathrm{ST}$ to the prospective nTS region is also normal in $R n x$ mutants (Fig. 5C,D, nTS). However, in marked contrast to the pattern seen in wild-type embryos (Fig. 5C,E), Rnx mutant afferents do not terminate in the prospective nTS area, but instead continue to grow dorsal-laterally into the adjacent territory (Fig. 5D,F, white arrows). Higher magnification shows that mutant axons directly cross the prospective target, rather than change the growth cone morphology and send side branches to ectopic sites (data not shown). The "overshooting" phenotype is fully consistent with a loss or a defect of the normal targets in the nTS. Moreover, no defect of neuronal differentiation in the primary sensory afferent neurons was detected, as indicated by normal expression of
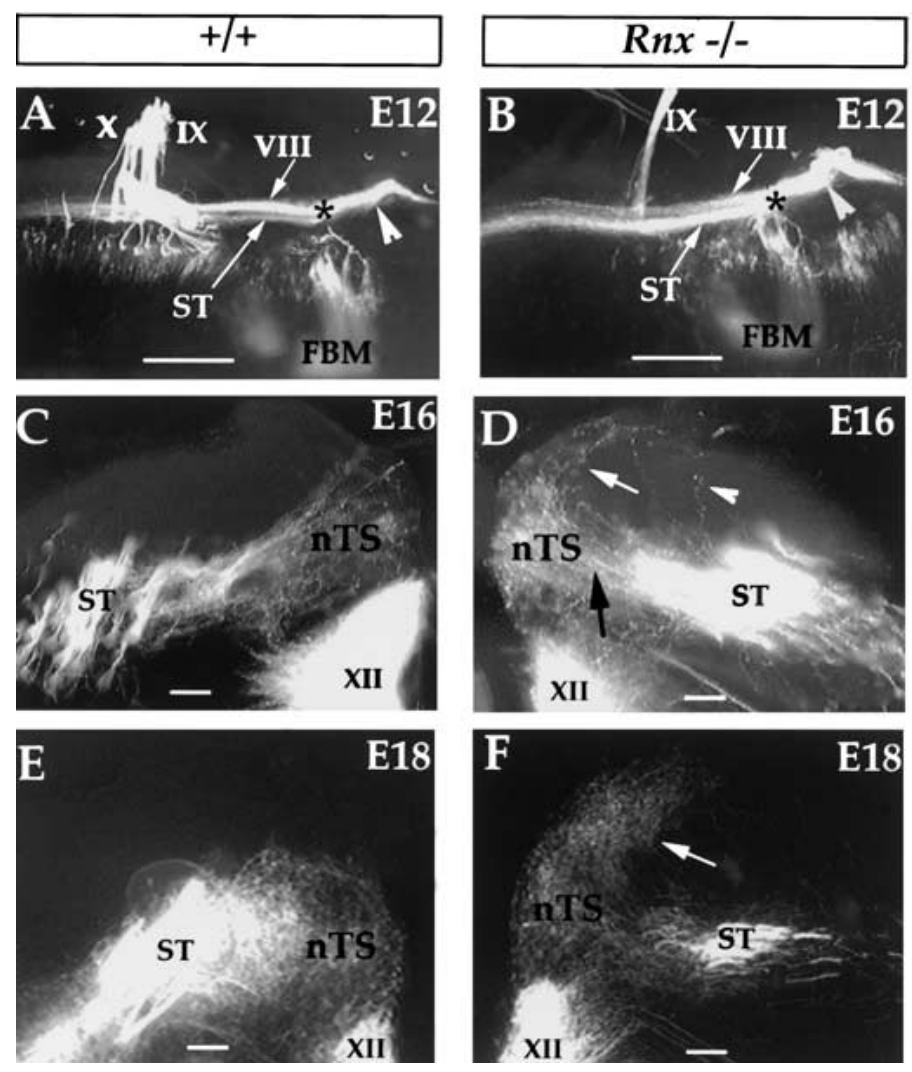
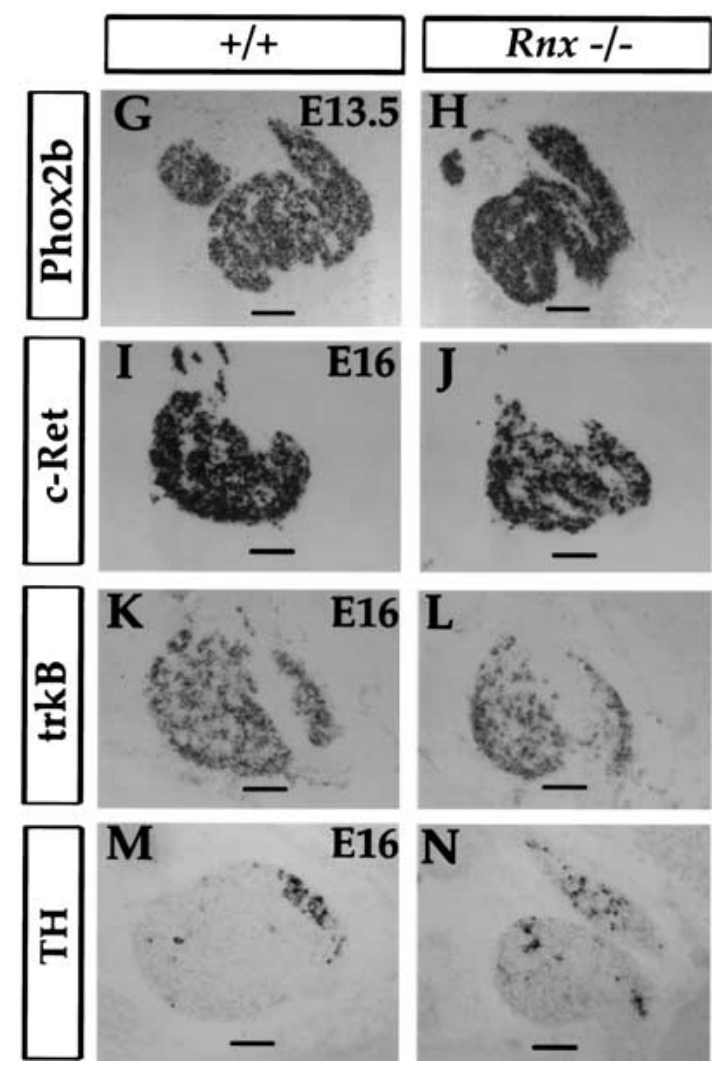

Figure 5. Aberrant central projection of visceral sensory afferents in Rnx mutants and normal development of peripheral visceral sensory ganglia. $(A, B)$ Flat-mounted E12 wild-type $(A)$ and $R n x$ mutant $(B)$ embryos. Dorsal is up and rostral is to the right. Central projections of the VIIth, VIIIth, IXth, and Xth nerves are labeled with DiI. (FBM) facial branchial motor nucleus. The axial position of FBM corresponds to the rostral limit of the descending solitary tract $\left(A, B,{ }^{\star}\right)$. Rostral to the FBM is the ascending VIIIth afferents $(A, B$, white arrowheads). Caudal to the FBM is the descending solitary tract (ST), which is located ventral to the descending VIII afferents $(A, B$, VIII). Clearly, the formation of the ST is not affected in Rnx mutants $(B, \mathrm{ST}) .(C-F)$ Transverse sections through the medulla of E16 and E18 wild-type $(C, E)$ and $R n x$ mutant embryos $(D, F)$, which are shown to mirror each other. Initial collateral projections from the ST to the prospective nTS area are not affected in Rnx mutants $(D$, black arrow). Only a very few aberrant projections from the ST are seen in $R n x$ mutants ( $D$, arrowhead). However, $R n x$ mutant afferents do not terminate in the area that normally constitutes the nTS. Instead they continue to grow dorsal-laterally to adjacent territory ( $D$, white arrow). By E18, most axons have passed through the prospective nTS area $(F$, arrow). $(G-N)$ Transverse sections through the nodose and pertrosal complex. In situ hybridization was performed with indicated probes and developmental stages. Note absence of any apparent alterations in expression patterns. Bars: $A, B$, $500 \mu \mathrm{m} ; C-F, 100 \mu \mathrm{m} ; G-N, 200 \mu \mathrm{m}$. 
a panel of markers including Phox2b (Pattyn et al. 1997), TH (Helke and Niederer 1990), DBH (Morin et al. 1997), trkB (Ernfors et al. 1992), and c-Ret (Morin et al. 1997; Pattyn et al. 1999) (Fig. 5G-N; data not shown).

Rnx is expressed transiently in, and required for proper formation of, the brainstem (nor)adrenergic centers

Phox $2 b$ is essential for formation of the (nor)adrenergic neurons throughout the nervous system (Pattyn et al. 2000a). Consistent with the loss of Phox $2 b$ expression in the nTS at late embryonic stages, the development of the A2/C2 cluster of the (nor)adrenergic neurons, which is located within the nTS, is compromised in Rnx mutants (Fig. 6C,D). The defect is indicated by a complete absence of expression of tyrosine hydroxylase $(T H)$ (data not shown) and dopamine-beta-hydroxylase $(D B H)$ (Fig. 6; data not shown), two key enzymes for catecholamine biosynthesis (Nagatsu et al. 1964; Friedman and Kaufman 1965). Surprisingly, formation of other (nor)adrenergic centers is also compromised in Rnx mutants, including A1/C1 (Fig. 6, cf. A and B), A5 (Fig. 6, cf. E and F), the locus subcoeruleus (sLC) (Fig. 6, cf. E and F), and the caudal part of the locus coeruleus (LC) (Fig. 6, cf. E and F, arrowhead). Only the most rostral part of the LC is still formed (Fig. 6, cf. E and F, LC).
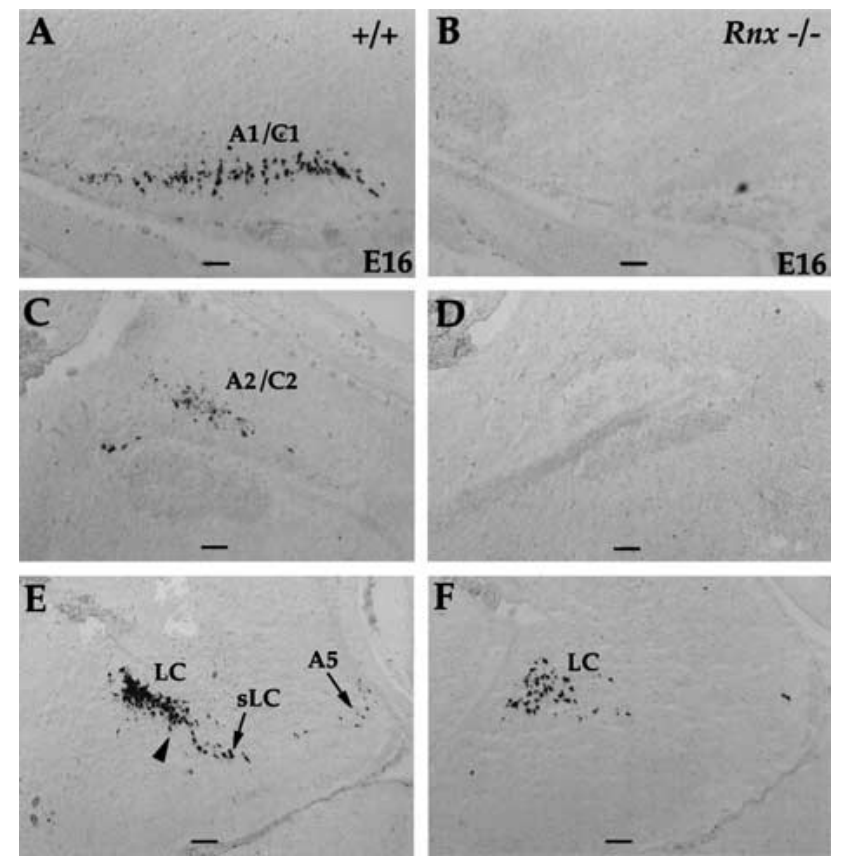

Figure 6. Formation of (nor)adrenergic neurons is compromised in $R n x$ mutants. Sagittal sections through E16 wild-type $(A, C, E)$ and $R n x$ mutant $(B, D, F)$ embryos. In situ hybridization was performed with $D B H$ probe. In $R n x$ mutants, expression of $D B H$ is absent in most of (nor)adrenergic centers, including $\mathrm{Al} /$ $\mathrm{C} 1$ (cf. $A$ and $B$ ), A2/C2 (cf. $C$ and $D$ ), A5 (cf. $E$ and $F$ ), locus subcoeruleus (sLC) (cf. $E$ and $F$ ), and caudal locus coeruleus (cf. $E$ and $F$, arrowhead). Only the rostral locus coeruleus is still formed in Rnx mutants (F, LC). Bars, $200 \mu \mathrm{m}$.
At E14.5 or later embryonic stages, no Rnx expression was detected in the (nor)adrenergic centers (data not shown). To determine whether $R n x$ is expressed transiently in these neurons, we examined Rnx expression at earlier embryonic stages. In the developing pons, (nor)adrenergic neurons are subdivided into two groups: (1) the LC, which is dependent on both Phox2 $a$ and Phox $2 b$ and develops from rhombomere 1 (Morin et al. 1997; Pattyn et al. 2000a, and (2) the A5 and the sLC, which require Phox $2 b$ function for the formation and develop from caudal developing pons (Morin et al. 1997; Pattyn et al. 2000a). Double staining shows that Rnx and Phox2a are coexpressed in the caudal but not the rostral part of developing pons of E10.5 wild-type embryos (Fig. 7A-E). Meanwhile, extensive coexpression of Phox $2 b$ and $R n x$ is detected in the most caudal part of developing pons (Fig. 7G,H). By E11.5, Rnx expression has been turned off in these Phox2a- or Phox $2 b$-expressing (nor)adrenergic neurons (Fig. 7F,I). Since expression of Phox2 genes is associated predominantly with (nor)adrenergic cells in developing pons (Pattyn et al. 2000a; also see below, Fig. 8 ), we conclude that $R n x$ is expressed transiently in subsets of prospective (nor)adrenergic neurons. Consistent with this, $D B H$ expression is eliminated in the caudal but not the rostral part of developing pons of E10.5 Rnx mutant embryos (Fig. 7J-O), which corresponds precisely to the partial defect seen in the pons area of E16.5 RnX mutants (Fig. 6E,F).

The development of medullary (nor)adrenergic neurons (the $\mathrm{A} 1 / \mathrm{C} 1$ and $\mathrm{A} 2 / \mathrm{C} 2$ clusters) occurs at later embryonic stages. $D B H$ expression in this area is first detected around E12.5, but is absent in Rnx mutants at all stages examined (data not shown). Therefore, $R n x$ is required for proper specification of (nor)adrenergic neurons throughout the developing hindbrain (except rostral locus coeruleus).

\section{Expression of Rnx and Phox $2 \mathrm{~b}$ may be independently regulated}

The dual genetic requirement of $R n x$ and Phox 2 genes in controlling $D B H$ expression raises two possible scenarios: (1) they function in a cascade, or (2) they function independently. Support for the latter scenario is that expression of Phox2 genes in prospective (nor)adrenergic neurons is independent of $R n x$. In caudal developing pons of both E10.5 and E11.5 wild-type embryos, all of the Phox $2 b$-expressing neurons have already turned on the expression of $D B H$ (Fig. 8E,G). Strikingly, while expression of $D B H$ is completely eliminated in E10.5 Rnx mutant embryos (Fig. 8A,B), neither Phox2b nor Phox2a expression is affected (Fig. 8, cf. C and D; data not shown). Selective elimination of $D B H$ expression is also observed in E11.5 mutant embryos (Fig. 8, cf. G and H).

Several observations also argue against the model that $R n x$ is a downstream target of Phox $2 b$. First, in the most dorsal part of developing medulla of E10.5 (data not shown) and E11.5 wild type embryos, expression of $R n x$ but not Phox $2 b$ is detected in cells that are close to the ventricular zone (Fig. 8I, arrows). In contrast, in 
a
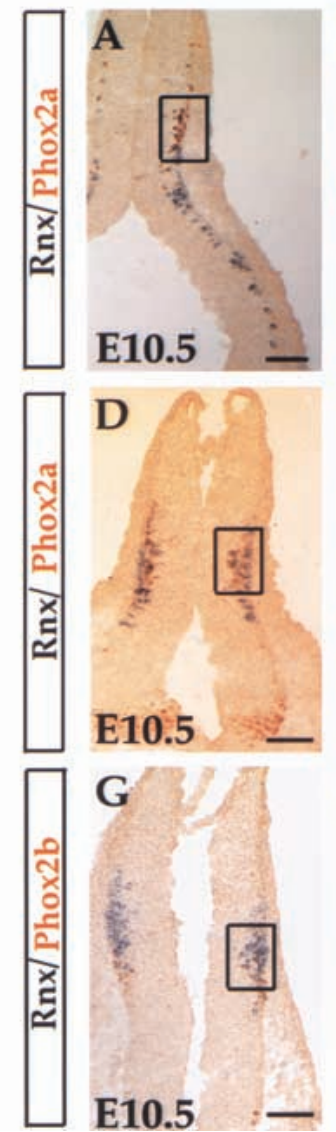
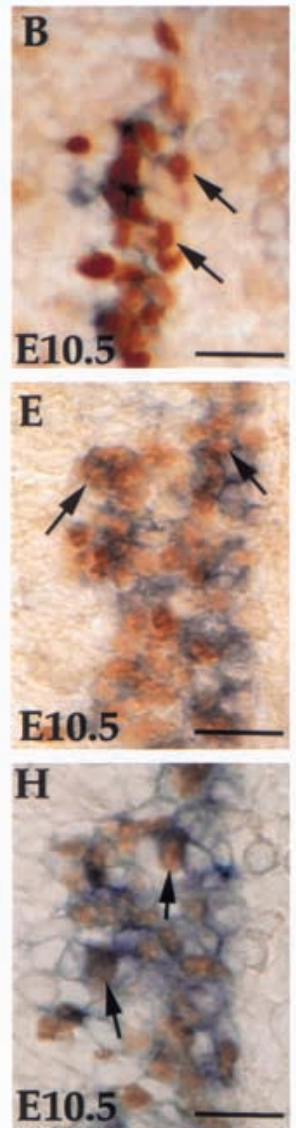
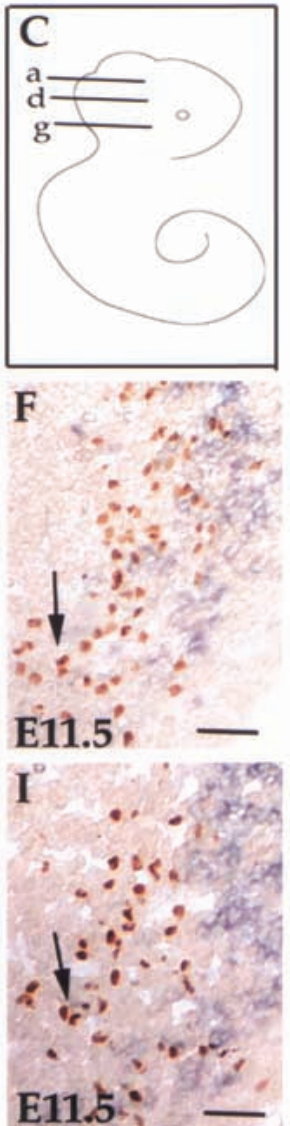

b
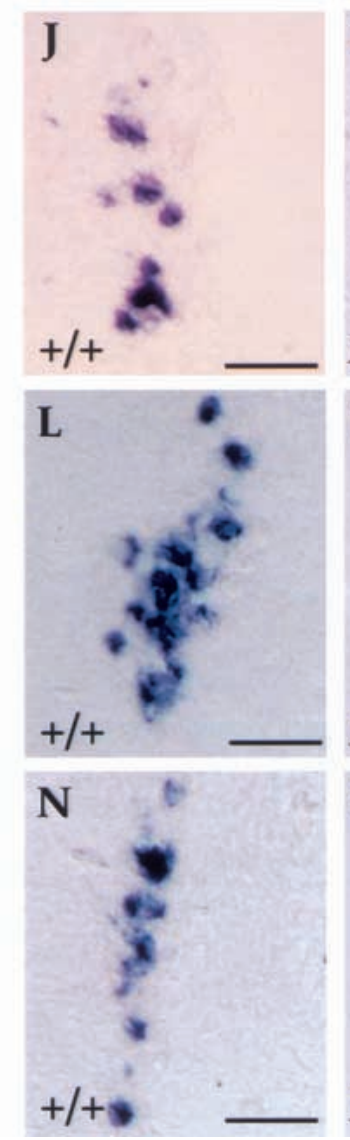

DBH
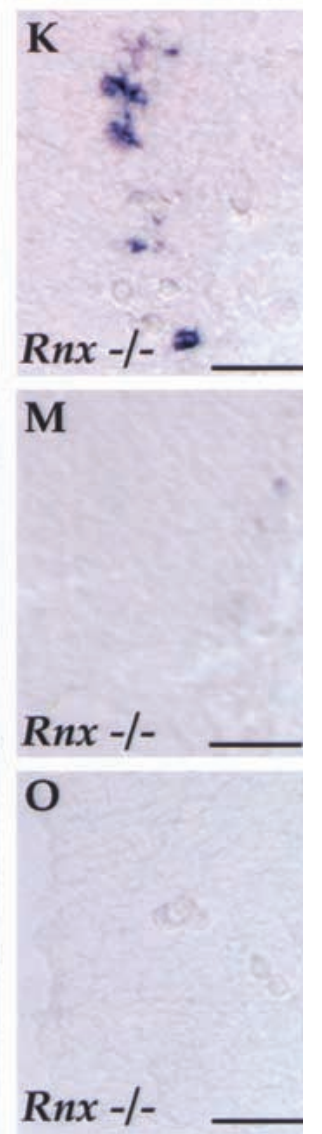

Figure 7. Overlapping expression of $R n x$ and Phox $2 a / P h o x 2 b$ in wild-type, and loss of $D B H$ expression in Rnx mutant developing pons. (a) Transverse sections through developing pons of wild-type embryos with indicated embryonic stages. Sections $A, D$, and $G$ are from three different axial levels of developing pons, corresponding to lines a, $\mathrm{d}$, and $\mathrm{g}$ of the cartoon shown in panel $C$, respectively. Lines a and $\mathrm{d}$ may correspond to rostral and caudal parts of rhombomere 1, respectively, whereas line g may correspond to rhombomere 2. Panels $B, E$, and $H$ are higher magnifications of the boxed regions shown in panels $A, D$, and $G$, respectively. Double staining $(R n x$ mRNA, purple; Phox 2 proteins, brown) shows that $R n x$ coexpresses with Phox $2 a$ in the caudal (E, arrows) but not rostral ( $B$, arrows) part of the developing pons. In the most caudal part of the developing pons, $R n x$ expression overlaps with that of $P h o x 2 b(H$, arrows). By E11.5, Rnx expression is turned off in both Phox2a-expressing cells $(F$, arrow) and Phox2b-expressing cells $(I$, arrow). (b) Transverse sections through developing pons of E10.5 wild-type $(J, L, N)$ and $R n x$ mutant embryos $(K, M, O)$. Panels $J$, $L$, and $N$ are roughly equivalent to panels $B, E$, and $H$, respectively. In situ hybridization was performed with $D B H$ probe. Expression of $D B H$ in the rostralmost part of developing pons was not affected (cf. $J$ and $K$ ), but is absent in the caudal parts of developing pons of E10.5 Rnx mutant embryos (cf. $L, N$ and $M, O)$. Bars: $A, D, G, 200 \mu \mathrm{m} ; B, E, H, 50 \mu \mathrm{m} ; F, I, 100 \mu \mathrm{m}$.; $J-O, 100 \mu \mathrm{m}$.

more matured marginal zone, all cells coexpress $R n x$ and Phox2b (Fig. 8I, arrowheads). Thus, Rnx expression precedes that of Phox $2 b$ in the cells that eventually coexpress both genes. Second, Rnx expression is not detected in many Phox $2 b$-expressing cells (see Fig. 2). Third, no Phox $2 b$ expression is detected in the trigeminal nuclei where $R n x$ is expressed (Y. Qian et al., unpubl.).

Besides Phox2b and Rnx, MASH1 is another transcription factor required for formation of brainstem (nor)adrenergic centers (Hirsch et al. 1998). Consistently, in E10.5 developing pons, $R n x$-expressing neurons are located precisely lateral to the portion of the ventricular zone that expresses MASH1 protein (Fig. 8J), suggesting that like the nTS, all (nor)adrenergic neurons develop from Mash1-positive precursors.

\section{Discussion}

Both relay visceral sensory and (nor)adrenergic neurons in the brainstem are critically important for cardiovascular and respiratory control. Prior to this study, formation of these neurons was poorly understood. Here we show that these two classes of neurons are ontogenetically related. Genetic analysis demonstrates that $R n x$ plays a central role in formation of these neurons (Fig. 9a). Importantly, we show that $R n x$ and Phox $2 b$ are independently required for specification of the (nor)adrenergic phenotype. Our findings suggest that network-spe- 

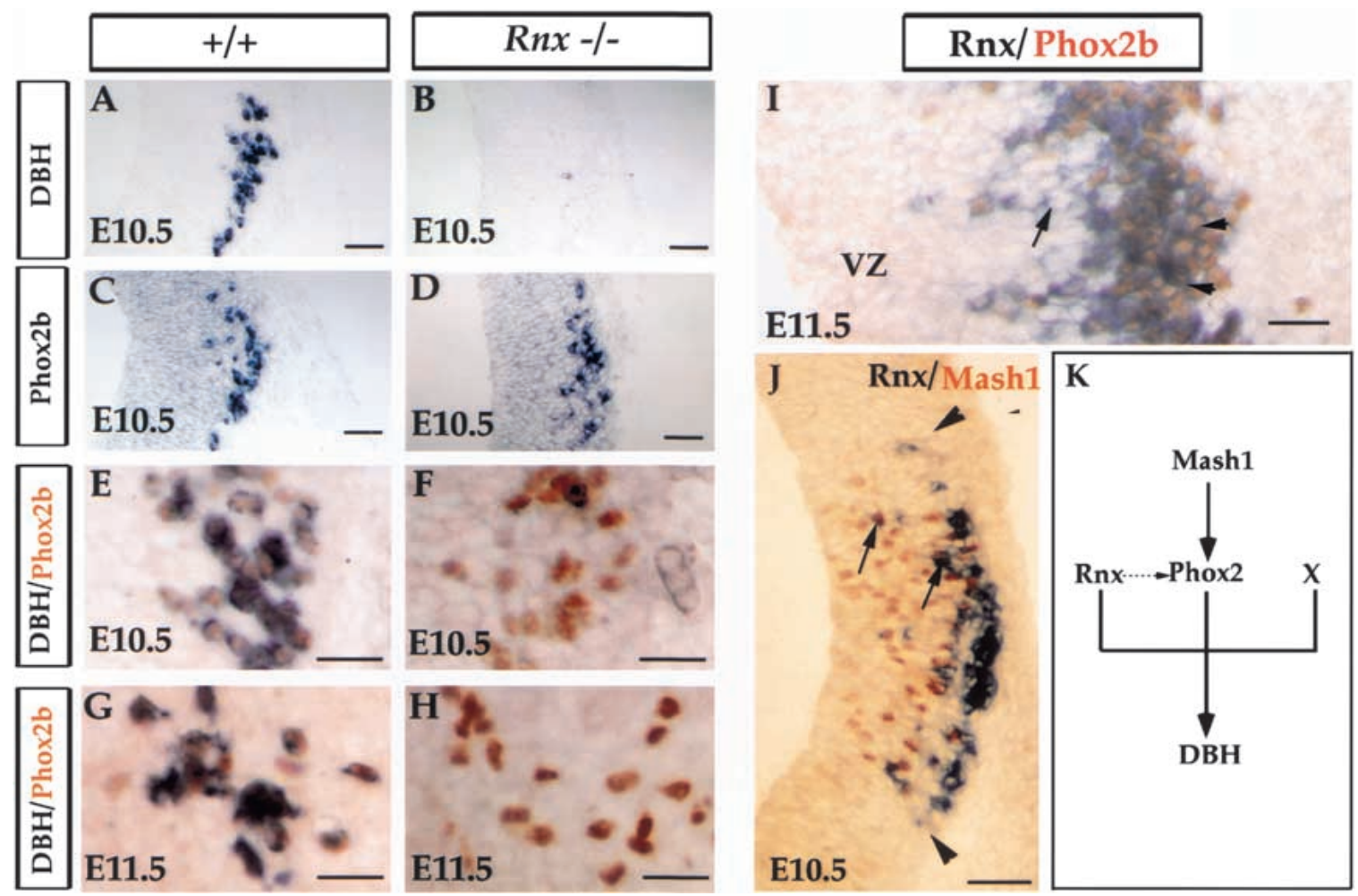

Figure 8. Independent regulation of Phox $2 b$ and $R n x$ expression. $(A-H)$ Transverse sections through caudal developing pons of E10.5 $(A-F)$ and E11.5 $(G, H)$ embryos. In situ hybridization was performed with indicated probes $(A-D)$. Panels $E-H$ show double labeling of $D B H$ mRNA (purple) and Phox2b protein (brown). $D B H$ and Phox2b are coexpressed in both E10.5 and E11.5 wild-type embryos (E, G). Although $D B H$ expression is absent in Rnx mutants (cf. $A$ and $B$ ), Phox $2 b$ expression is still detected in both E10.5 (cf. $D, F$ and $C, E$ ) and E11.5 (cf. $H$ and $G$ ) Rnx mutants. (I) Longitudinal section through the dorsal stripe of Rnx-expressing cells of E11.5 wild-type embryos. Double staining of $R n x$ mRNA (purple) and Phox2b protein (brown). In a region closer to the ventricular zone (VZ), many cells express $R n x$ but not Phox $2 \mathrm{~b}$ (I, arrows). In contrast, coexpression of these two genes was detected throughout the marginal zone (I, arrowheads). ( $J$ ) Double staining of Rnx mRNA (purple) and MASH1 protein (brown) on transverse sections through developing pons of E10.5 wild-type embryos. The axial level corresponds to the sections shown in panels $A-D$. Rnx-expressing cells are located lateral to the Mash1-expressing domain, and they share identical boundaries along the dorsal ventral axis $(J$, arrowheads). A few doublestained cells were detected ( $J$, arrows). ( $K$ ) Schematic summary diagram regarding $D B H$ regulation in brainstem (nor)adrenergic neurons. MASH1 and Phox 2 proteins function in a cascade, whereas Rnx and Phox $2 \mathrm{~b}$ may function independently in regulating $D B H$ expression. The dashed arrow is a hypothetical function of $\mathrm{Rnx}$ in maintaining Phox2 expression at later developmental stages. $\mathrm{X}$ is a hypothetical regulator. See text for detailed discussion. Bars: $A-D, 100 \mu \mathrm{m} ; E-I, 50 \mu \mathrm{m} ;, 100 \mu \mathrm{m}$.

cific transcription factors may be a common theme in developing nervous system.

\section{The developmental origin, and a requirement of Rnx for formation, of first-order relay visceral sensory neurons}

The studies reported here establish $R n x$ as the first gene characterized thus far that is required for proper formation of first-order relay visceral sensory neurons in the medulla (the nTS and area postrema). The defect of the nTS is first indicated by a complete loss of Phox $2 b$ expression at late embryonic stages. Examination of markers (Phox $2 b$ and Ebf2) expressed in the surrounding region shows that the whole nTS structure seems to be missing. The defect is further supported by aberrant central projections of the primary visceral sensory afferents, since no abnormality of neuronal differentiation is de- tected in primary visceral sensory ganglia. The restriction of defect to central visceral sensory neurons is consistent with the finding that among the three Tlx family members, only $R n x$ is expressed in the nTS, whereas both Enx and Rnx are expressed in the peripheral ganglia and might therefore have redundant or compensating functions there (data not shown).

Our studies reveal a possible embryological origin for the nTS neurons. We find that of the two longitudinal stripes of Rnx-expressing cells in the developing medulla, only dorsally derived neurons coexpress $R n x$ and Phox $2 b$. These double-positive cells migrate ventrally, and aggregate together to form the nTS (and part of (norladrenergic neurons; see below; Fig. 9b). Furthermore, the nTS neurons are apparently derived from the ventricular zone corresponding to the most dorsal part of the Mash1-expressing domain (Fig. 9b). Our conclusion is in contrast to the previous hypothesis that the nTS devel- 
Defects in Rnx Mutants

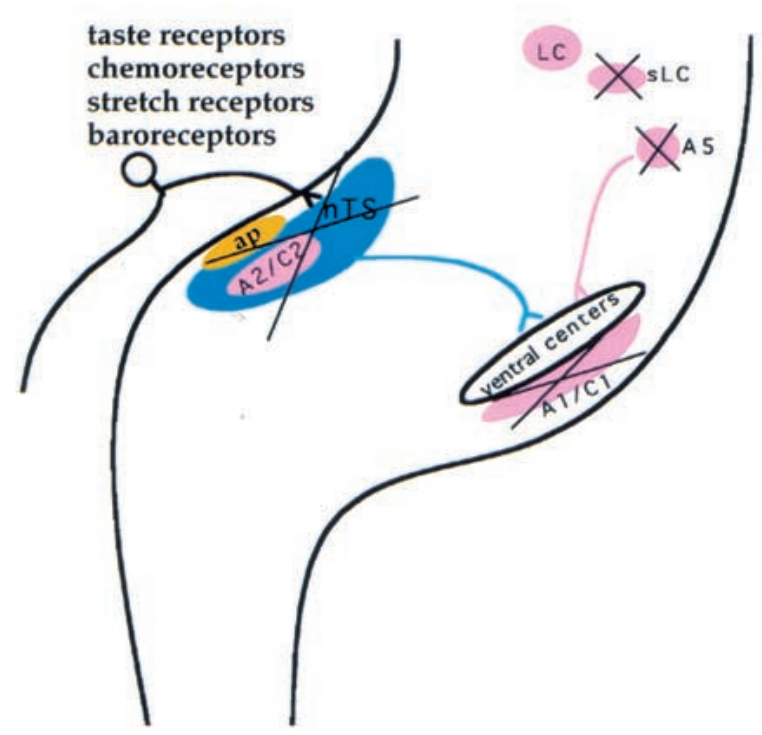

b

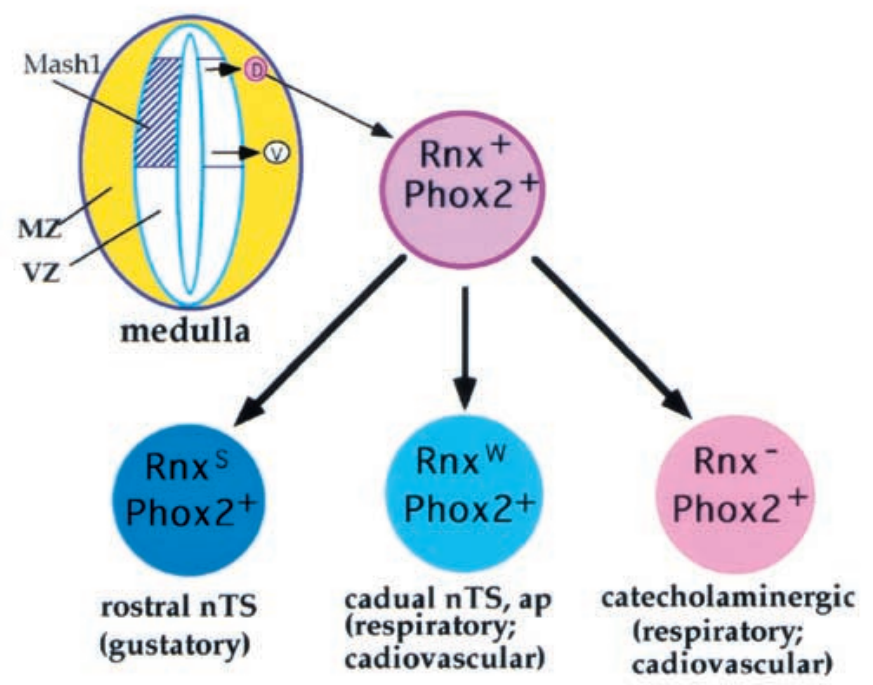

Figure 9. (a) Summary of developmental defects in Rnx mutants. The formation of the first-order relay visceral sensory neurons in the nTS and the area postrema (ap), and most of the (nor)adrenergic centers (pink), is compromised in Rnx mutants. Only a subset of (nor)adrenergic neurons in the locus coeruleus (LC) still forms. The nTS and the ap receive innervation of various visceral sensory afferents, including taste receptors, baroreceptors, chemoreceptors, and pulmonary stretch receptors. The defects of these autonomic neuronal structures not only explain why Rnx mutant mice suffer from breathing problems, but also indicate that the mutant mice may have additional problems in the regulation of cardiovascular and gustatory functions. $(b)$ Ontogeny of relay visceral sensory and (nor)adrenergic neurons. Both groups of neurons develop from the precursors expressing Mash1, and they coexpress Rnx and Phox $2 b$ (or Phox2a) when they are formed. In the case of the developing medulla, like the nTS, the (nor)adrenergic neurons may develop from dorsally derived $R n x$-expressing cells (pink circle, D), although we have not excluded the possibility that ventrally derived Rnxexpressing cells (white circle, V) may give rise to the ventral Al/Cl cluster of (nor)adrenergic neurons (see text for detailed discussion). It is interesting to note that strong $R n x$ expression $\left(\mathrm{Rnx}^{\mathrm{S}}\right)$ is sustained in rostral nTS, which is required for gustatory function. In contrast, $R n x$ expression is either reduced $\left(\mathrm{Rnx}^{\mathrm{W}}\right)$ in caudal $\mathrm{nTS}$ and the area postrema (ap), or is turned off completely in (nor)adrenergic neurons, both of which are associated with cardiovascular and respiratory control. VZ, the ventricular zone where dividing precursors are located; MZ, the marginal zone where postmitotic neurons are located.

ops from the ventral stripe of Rnx-expressing cells in chick embryos, which is primarily based on Rnx expression at early embryonic stages (Logan et al. 1998).

Rnx is required for specification of (nor)adrenergic neurons

Besides MASH1 and Phox2 proteins, Rnx is the third transcription factor required for formation of the majority of brainstem (nor)adrenergic neurons, as indicated by the absence of $D B H$ expression in Rnx-deficient mice. Our studies suggest possible origins of the (nor)adrenergic neurons. In developing pons, (nor)adrenergic neurons, including sublocus coeruleus and the A5/A7 clusters, can be prospectively defined by combinatorial expression of Rnx and Phox2a/Phox2b, and these neurons develop from an intermediate portion of the ventricular zone along the dorsal-ventral axis, in which Mash1 is expressed (Fig. 8J). The formation of medullary (nor)adrenergic neurons (the $\mathrm{A} 1 / \mathrm{C} 1$ and $\mathrm{A} 2 / \mathrm{C} 2$ clusters) depends on Phox2b (Pattyn et al. 2000a), but not on Phox2a (Morin et al. 1997). There are two stripes of $R n x$-expressing cells in the developing medulla. Since only dorsally derived cells coexpress Rnx and Phox $2 b$ (Fig. 2), the dual genetic requirement of these two genes for the formation of (nor)adrenergic neurons suggests that these neurons develop from dorsally derived Rnx-expressing cells, as the neurons in the nTS do (Fig. 9b). Support for this is that the A2/C2 cluster is located within the nTS (Fig. 1). We have not yet excluded the possibility that ventrally derived Rnx-expressing cells might give rise to the ventral A1/Cl cluster of (nor)adrenergic neurons. However, this possibility assumes that $P$ hox $2 b$ expression is initiated after Rnx expression has been turned off, because no coexpression of $R n x$ and Phox $2 b$ is detected in ventrally derived cells (Fig. 2).

Rnx might be the missing factor that functions cooperatively with Phox $2 b$ in specification of the (nor)adrenergic phenotype, because these two genes are apparently independently regulated. First, $R n x$ expression precedes that of Phox $2 b$ in cells that eventually coexpress both genes (Fig. 8I). Indeed, Rnx expression in these doublepositive cells is not affected in the Phox2b mutant (J.F. Brunet, pers. comm.). Second, initial expression of $P h o x 2 b$ in prospective (nor)adrenergic neurons (and relay visceral sensory neurons) is not affected in Rnx-deficient 
mice (Figs. 4,8). However, it should be pointed out that $R n x$ and Phox $2 b$ are coexpressed in non-(nor)adrenergic neurons in the nTS, suggesting that specification of the (nor)adrenergic phenotype may require additional factors (Fig. 8K). It is also important to note that Phox $2 b$ expression in prospective (nor)adrenergic neurons and relay visceral sensory neurons is eventually lost in $R n x$-deficient mice, suggesting that either the mutant cells die or Rnx has a later role in maintaining Phox2 expression (Fig. 8J, dashed arrow). Support for a cell loss model is that the $\mathrm{nTS}$ structure is apparently missing in $R n x$ mutants (Fig. 4E-H).

\section{Rnx deficiency and failure of respiratory control}

$R n x$-deficient mice die from respiratory failure (Shirasawa et al. 2000). Electrophysiological recordings show a rapid respiratory rate and frequent apneas in $R n x$ mutant mice (Shirasawa et al. 2000). The observation that $R n x$ is expressed in the area dorsal to the nucleus ambiguus (nA) whereas respiratory neurons are located ventral to the nA (Rekling and Feldman 1998) suggests that the development of rhythmic respiratory neurons per se may not be affected in Rnx-deficient mice. Consistent with this, expression of neurokinin-1 receptor, a possible anatomical marker for pacemaker respiratory neurons (Gray et al. 1999), is not affected in Rnx-deficient mice.

The developmental defects of the nTS, the area postrema, and the (nor)adrenergic neurons offer an alternative explanation. In wild-type mice, input from noradrenergic neurons causes a depression of respiratory frequency (Errchidi et al. 1991; Al-Zubaidy et al. 1996). Therefore, the improper formation of noradrenergic neurons might contribute to the rapid shallow breathing symptom observed in $R n x$-deficient mice (Shirasawa et al. 2000). Transection of the chemoreceptor afferents in neonatal rat (Hofer 1986), or a loss of peripheral visceral sensory neurons as in BDNF-deficient mice (Erickson et al. 1996; Brady et al. 1999), causes irregular respiratory rhythms. Because the nTS and the area postrema contain first-order relay neurons for all of the visceral sensory afferents, the apparent loss of these two structures in $R n x$-deficient mice should cause a disorder in respiratory control. Moreover, the mutant mice may suffer additional physiological problems, such as defect of taste sensation and abnormality of cardiovascular control (Fig. 9a).

\section{Network-specific transcription factors and formation of neuronal circuitry}

Recent studies show that Math1, which encodes a basic helix-loop-helix type of transcription factor, is required for the formation of most components of the proprioceptive neuronal circuitry (Bermingham et al. 2001). The concept of network-specific transcription factors was first suggested by Brunet and his colleagues, based on Phox2 gene expression in and requirement for the formation of many components within autonomic neuronal circuitry (Tiveron et al. 1996; Morin et al. 1997; Pattyn 1997, 1999, 2000a). However, Phox2b is also associated with several nonautonomic structures, such as the branchial motor neurons (Pattyn et al. 2000b) and a large group of uncharacterized neurons in the medulla (Fig. 2F, red arrow).

Thus, it is important to emphasize that the combinatorial expression of Rnx and Phox $2 b / P h o x 2 a$ is restricted exclusively to autonomic components, including the $\mathrm{nTS}$, the area postrema, the (nor)adrenergic centers, the peripheral visceral sensory ganglia (Fig. 2A,C, g, p, and $\mathrm{n})$, and the sympathetic and parasympathetic ganglia (data not shown). Most strikingly, most of these autonomic components develop from Mash1-positive precursors (Fig. 9b; Anderson et al. 1997). (The visceral sensory ganglia are the only exception, and are derived from neurogenin2-positive placodal neural precursors; Fode et al. 1998). Thus, it appears to be an emerging common theme that the same set of regulatory molecules is repeatedly involved in the development of multiple components within a physiological neuronal network. Future studies will be directed to determine whether or not network-specific transcription factors control the expression of network-specific molecules, such as members of the cadherin superfamily (Suzuki et al. 1997; Inoue et al. 1998; Yagi and Takeichi 2000).

\section{Materials and methods}

\section{Animals}

The generation of ngn1 and Rnx mutant mice has been described (Ma et al. 1998; Shirasawa et al. 2000). The morning that vaginal plugs were observed was considered E0.5. PCR-based genotyping of ngn1 mutant mice was performed as described (Ma et al. 2000). The mutant Rnx allele was detected by PCR amplification with primers derived from the neo gene (Ma et al. 2000), which produces a $0.6 \mathrm{~kb}$ product. The wild-type $R n x$ allele was detected by the following PCR primers: 5 '-AGCG GCGACTGCTCTCCATCCAGG-3' and 5'-GCATCGACCAA ATCCTCAACAGCC-3', which produces a $0.3 \mathrm{~kb}$ product.

\section{In situ hybridization}

Section and whole-mount in situ hybridizations were performed as described (Ma et al. 1998). In situ hybridization combined with Phox $2 b$ antibody immunostaining was performed essentially as described (Tiveron et al. 1996). The Tlx1 probe $(0.7 \mathrm{~kb})$, the Enx probe $(1.0 \mathrm{~kb})$, and the $R n x$ probe $(1.0 \mathrm{~kb})$ were amplified by RT-PCR using a cDNA template prepared from the trigeminal ganglia of E12.5 mouse embryos. Other probes used in this study were Phox2a (Tiveron et al. 1996), Phox2b (Pattyn et al. 1997), TH (Pattyn et al. 2000), DBH (Morin et al. 1997), trkB (Birren et al. 1993), and c-Ret (Morin et al. 1997).

\section{TUNEL staining on cryostat sections}

Three pairs of E10.5-E19 wild-type and mutant embryos were used. TUNEL staining on frozen sections was performed by the ApopTag method according to the manufacturer's instructions (Intergen). 


\section{In situ hybridization combined with Brdu immunostaining}

For transient labeling, pregnant wild-type mice carrying E11.5 embryos were injected with 5-bromo-2-deoxy-uridine (BrdU) (Sigma) (50 mg/kg body weight) $2 \mathrm{~h}$ before they were sacrificed. For pulse-chase labeling, BrdU was injected at E10.5 and collected at E12.5. In situ hybridization was performed first, followed by immunostaining with anti-BrdU antibody. After in situ hybridization, sections were first washed with PBS $(3 \times 5$ $\mathrm{min})$, fixed in $4 \%$ paraformaldehyde for $15 \mathrm{~min}$, washed with PBS $(3 \times 5 \mathrm{~min})$, treated with $2 \mathrm{~N} \mathrm{HCl}$ in PBS for $30 \mathrm{~min}$ at $37^{\circ} \mathrm{C}$, rinsed in PBS five times, and washed with PBS $(3 \times 5 \mathrm{~min})$. The subsequent immunostaining with anti-BrdU antibody (Boehringer Mannheim) (1:50 dilution) was performed with a Vectastain detection kit (Vector labs).

\section{DiI tracing}

E12 embryos for DiI-labeling were directly fixed by immersing them in 4\% paraformaldehyde. E14-E18 embryos were first perfused with and then immersed in $4 \%$ paraformaldehyde. Two to four pairs of wild-type and mutant embryos were used. For E12 wild-type and Rnx mutant embryos, DiI was injected into the facial and vestibular-cochlear complex as well as the IXth and Xth nerves. For each of the embryos at E14 or later stages, DiI was applied to the facial and vestibular-cochlear complex on one side of the embryos, and to the IXth and Xth nerves on the contralateral side. DiI was soaked in filter (Fritzsch and Nichols 1993) and diffused for three (E12 embryos), four (E14-E16 embryos), or five days (E17 and E18 embryos) at $37^{\circ} \mathrm{C}$. Brains of E12 mice were dissected, mounted flat, and viewed with an epifluorescence microscope. Brains of the E14-E18 embryos and newborn mice were embedded in $12 \%$ gelatin and cut with a vibratome at $80 \mu \mathrm{m}$ thickness. Sections were mounted and viewed with an epifluorescence microscope and a Biorad Radiance 2000 confocal microscope.

\section{Acknowledgments}

Our special thanks go to Dr. Stanley Korsmeyer for providing the Rnx knockout mice, Dr. Jean-François Brunet for the Phox $2 \mathrm{a}$ and Phox $2 \mathrm{~b}$ antibodies and for sharing unpublished data, and Li-Ching Lo and David J. Anderson for the MASH1 antibody. We thank Drs. Jean-François Brunet, David Rowitch, Jeremy Green, Xi He, Paul Gray, Rhonda Hyde, and anonymous reviewers for critical comments on the manuscript. Q.M. is a Claudia Adams Barr Investigator and a Pew Scholar in Biomedical Sciences. This work was supported in part by the NIH grants 1 R01 DE13843-01 (Q.M.) and 2 P01 DC 0015 (B.F.).

The publication costs of this article were defrayed in part by payment of page charges. This article must therefore be hereby marked "advertisement" in accordance with 18 USC section 1734 solely to indicate this fact.

\section{References}

Al-Zubaidy, Z.A., Erickson, R.L., and Greer, J.J. 1996. Serotonergic and noradrenergic effects on respiratory neural discharge in the medullary slice preparation of neonatal rats. Pflugers Arch. 431: 942-949.

Altman, J. and Bayer, S.A. 1987. Development of the precerebellar nuclei in the rat: IV. The anterior precerebellar extramural migratory stream and the nucleus reticularis tegmenti pontis and the basal pontine gray. J. Comp. Neurol. 257: 529-552.

Anderson, D.J., Groves, A.K., Lo, L., Ma, Q., Rao, M., Shah, N.M., and Sommer, L. 1997. Cell lineage determination and the control of neuronal identity in the neural crest. Cold Spring Harbor Symp. Quant. Biol. 62: 493-504.
Bermingham, N.A., Hassan, B.A., Wang, V.Y., Fernandez, M., Banfi, S., Bellen, H.J., Fritzsch, B., and Zoghbi, H. Y. 2001. Proprioceptor pathway development is dependent on math1. Neuron 30: 411-422.

Birren, S.J., Lo, L.C., and Anderson, D.J. 1993. Sympathetic neurons undergo a developmental switch in trophic dependence. Development 119: 597-610.

Blessing, W.W. 1997. The lower brainstem and body homeostasis. Oxford University Press, New York, NY.

Bolme, P. and Fuxe, K. 1973. Pharmacological studies on a possible role of central noradrenaline neurons in respiratory control. J. Pharm. Pharmacol. 25: 351-352.

Brady, R., Zaidi, S.I., Mayer, C., and Katz, D.M. 1999. BDNF is a target-derived survival factor for arterial baroreceptor and chemoafferent primary sensory neurons. I. Neurosci. 19: 2131-2142.

Champagnat, J., Denavit-Saubie, M., Henry, J.L., and Leviel, V. 1979. Catecholaminergic depressant effects on bulbar respiratory mechanisms. Brain Res. 160: 57-68.

Dear, T.N., Sanchez-Garcia, I., and Rabbitts, T.H. 1993. The HOX11 gene encodes a DNA-binding nuclear transcription factor belonging to a distinct family of homeobox genes. Proc. Nat1. Acad. Sci. 90: 4431-4435.

Dear, T.N., Colledge, W.H., Carlton, M.B., Lavenir, I., Larson, T., Smith, A.J., Warren, A.J., Evans, M.J., Sofroniew, M.V., and Rabbitts, T.H. 1995. The Hox11 gene is essential for cell survival during spleen development. Development 121: 2909-2915.

Dube, I.D., Kamel-Reid, S., Yuan, C.C., Lu, M., Wu, X., Corpus, G., Raimondi, S.C., Crist, W.M., Carroll, A.J., Minowada, J., et al. 1991. A novel human homeobox gene lies at the chromosome 10 breakpoint in lymphoid neoplasias with chromosomal translocation $\mathrm{t}(10 ; 14)$. Blood 78: 2996-3003.

Erickson, J.T., Conover, J.C., Borday, V., Champagnat, J., Barbacid, M., Yancopoulos, G., and Katz, D.M. 1996. Mice lacking brain-derived neurotrophic factor exhibit visceral sensory neuron losses distinct from mice lacking NT4 and display a severe developmental deficit in control of breathing. $J$. Neurosci. 16: 5361-5371.

Ernfors, P., Merlio, J.-P., and Persson, H. 1992. Cells expressing mRNA for neurotrophins and their receptors during embryonic rat development. Eur. J. Neurosci. 4: 1140-1158.

Errchidi, S., Monteau, R., and Hilaire, G. 1991. Noradrenergic modulation of the medullary respiratory rhythm generator in the newborn rat: An in vitro study. J. Physiol. 443: 477-498.

Fode, C., Gradwohl, G., Morin, X., Dierich, A., LeMeur, M., Goridis, C., and Guillemot, F. 1998. The bHLH protein NEUROGENIN 2 is a determination factor for epibranchial placode-derived sensory neurons. Neuron 20: 483-494.

Friedman, S. and Kaufman, S. 1965. 3,4-dihydroxyphenylethylamine beta-hydroxylase. Physical properties, copper content, and role of copper in the catalytic activity. I. Biol. Chem. 240: 4763-4773.

Fritzsch, B. and Nichols, D.H. 1993. DiI reveals a prenatal arrival of efferents at the differentiating otocyst of mice. Hear. Res. 65: 51-60.

Garel, S., Marin, F., Mattei, M.G., Vesque, C., Vincent, A., and Charnay, P. 1997. Family of Ebf/Olf-1-related genes potentially involved in neuronal differentiation and regional specification in the central nervous system. Dev. Dyn. 210: 191-205.

Gavrieli, Y., Sherman, Y., and Ben-Sasson, S. A. 1992. Identification of programmed cell death in situ via specific labeling of nuclear DNA fragmentation. J. Cell Biol. 119: 493-501.

Gowan, K., Helms, A.W., Hunsaker, T.L., Collisson, T., Ebert, P.J., Odom, R., and Johnson, J.E. 2001. Crossinhibitory activities of Ngn1 and Math1 allow specification of distinct 
dorsal interneurons. Neuron 31: 219-232.

Gradwohl, G., Fode, C., and Guillemot, F. 1996. Restricted expression of a novel murine atonal-related bHLH protein in undifferentiated neural precursors. Dev. Biol. 180: 227-241.

Gray, P.A., Rekling, J.C., Bocchiaro, C.M., and Feldman, J.L. 1999. Modulation of respiratory frequency by peptidergic input to rhythmogenic neurons in the preBotzinger complex. Science 286: 1566-1568.

Guo, S., Brush, J., Teraoka, H., Goddard, A., Wilson, S.W., Mullins, M.C., and Rosenthal, A. 1999. Development of noradrenergic neurons in the zebrafish hindbrain requires BMP, FGF8, and the homeodomain protein soulless/Phox2a. Neuron 24: 555-566.

Hatano, M., Roberts, C.W., Minden, M., Crist, W.M., and Korsmeyer, S.J. 1991. Deregulation of a homeobox gene, HOX11, by the $\mathrm{t}(10 ; 14)$ in $\mathrm{T}$ cell leukemia. Science 253: 79-82.

Hatano, M., Aoki, T., Dezawa, M., Yusa, S., Iitsuka, Y., Koseki, H., Taniguchi, M., and Tokuhisa, T. 1997. A novel pathogenesis of megacolon in Ncx/Hox11L.1 deficient mice. J. Clin. Invest. 100: 795-801.

Helke, C.J. and Niederer, A.J. 1990. Studies on the coexistence of substance P with other putative transmitters in the nodose and petrosal ganglia. Synapse 5: 144-151.

Hirsch, M.R., Tiveron, M.C., Guillemot, F., Brunet, J.F., and Goridis, C. 1998. Control of noradrenergic differentiation and Phox2a expression by MASH1 in the central and peripheral nervous system. Development 125: 599-608.

Hofer, M.A. 1986. Role of carotid sinus and aortic nerves in respiratory control of infant rats. Am. J. Physiol. 251: R811-R817.

Inoue, T., Tanaka, T., Suzuki, S.C., and Takeichi, M. 1998. Cadherin-6 in the developing mouse brain: Expression along restricted connection systems and synaptic localization suggest a potential role in neuronal circuitry. Dev. Dyn. 211: 338-351.

Kennedy, M.A., Gonzalez-Sarmiento, R., Kees, U.R., Lampert, F., Dear, N., Boehm, T., and Rabbitts, T.H. 1991. HOX11, a homeobox-containing T-cell oncogene on human chromosome 10q24. Proc. Nat1. Acad. Sci. 88: 8900-8904.

Kim, H.S., Hong, S.J., LeDoux, M.S., and Kim, K.S. 2001. Regulation of the tyrosine hydroxylase and dopamine beta-hydroxylase genes by the transcription factor AP-2. I. Neurochem. 76: 280-294.

Lo, L., Morin, X., Brunet, J.-F., and Anderson, D.J. 1999. Specification of neurotransmitter identity by Phox 2 proteins in neural crest stem cells. Neuron 22: 693-705.

Logan, C., Wingate, R.J.T., McKay, I.J., and Lumsden, A. 1998. TlX-1 and TlX-3 homeobox gene expression in cranial sensory ganglia and hindbrain of the chick embryo: Markers of patterned connectivity. J. Neurosci. 18: 5389-5402.

Ma, Q., Sommer, L., Cserjesi, P., and Anderson, D.J. 1997. Mash1 and neurogenin1 expression patterns define complementary domains of neuroepithelium in the developing CNS and are correlated with regions expressing Notch ligands. $J$. Neurosci. 17: 3644-3652.

Ma, Q., Chen, Z.F., Barrantes, I.B., de la Pompa, J.L., and Anderson, D.J. 1998. Neurogenin 1 is essential for the determination of neuronal precursors for proximal cranial sensory ganglia. Neuron 20: 469-482.

Ma, Q., Anderson, D.J., and Fritzsch, B. 2000. Neurogenin1 null mutant ears develop fewer, morphologically normal hair cells in smaller sensory epithelia devoid of innervation. $J$. Assoc. Res. Otolaryngol. 1: 129-143.

Morin, X., Cremer, H., Hirsch, M.-R., Kapur, R.P., Goridis, C., and Brunet, J.-F. 1997. Defects in sensory and autonomic ganglia and absence of locus coeruleus in mice deficient for the homeobox gene Phox2a. Neuron 18: 411-423.

Nagatsu, T., Levitt, M., and Udenfriend, S. 1964. Tyrosine hy- droxylase, the initial step in norepinephrine biosynthesis. J. Biol. Chem. 239: 2910-2917.

Pattyn, A., Morin, X., Cremer, H., Goridis, C., and Brunet, J.-F. 1997. Expression and interactions of the two closely related homeobox genes Phox $2 a$ and Phox2b during neurogenesis. Development 124: 4065-4075.

Pattyn, A., Morin, X., Cremer, H., Goridis, C., and Brunet, J.-F. 1999. The homeobox gene Phox $2 b$ is essential for the development of autonomic neural crest derivatives. Nature 399: 366-370.

Pattyn, A., Goridis, C., and Brunet, J.F. 2000a. Specification of the central noradrenergic phenotype by the homeobox gene Phox2b. Mol. Cell Neurosci. 15: 235-243.

Pattyn, A., Hirsch, M., Goridis, C., and Brunet, J.F. 2000b. Control of hindbrain motor neuron differentiation by the homeobox gene Phox2b. Development 127: 1349-1358.

Raju, K., Tang, S., Dube, I.D., Kamel-Reid, S., Bryce, D.M., and Breitman, M.L. 1993. Characterization and developmental expression of Tlx-1, the murine homolog of HOX11. Mech. Dev. 44: 51-64.

Rekling, J.C. and Feldman, J.L. 1998. PreBotzinger complex and pacemaker neurons: Hypothesized site and kernel for respiratory rhythm generation. Annu. Rev. Physiol. 60: 385-405.

Roberts, C.W., Shutter, J.R., and Korsmeyer, S.J. 1994. Hox11 controls the genesis of the spleen. Nature 368: 747-749.

Roberts, C.W., Sonde, A.M., Lumsden, A., and Korsmeyer, S.J. 1995. Development expression of Hox 11 and specification of splenic cell fate. Am. J. Pathol. 146: 1089-1101.

Saper, C.B. 2000. Brain stem, reflexive behavior, and the cranial nerves. In Principles of neural science (eds. E.R. Kandel, J.H. Schwartz and T.M. Jessell), pp. 873-888. McGraw-Hill, New York, NY.

Shirasawa, S., Yunker, A.M., Roth, K.A., Brown, G.A., Horning, S., and Korsmeyer, S.J. 1997. Enx (Hox11L1)-deficient mice develop myenteric neuronal hyperplasia and megacolon. Nat. Med. 3: 646-650.

Shirasawa, S., Arata, A., Onimaru, H., Roth, K.A., Brown, G.A., Horning, S., Arata, S., Okumura, K., Sasazuki, T., and Korsmeyer, S.J. 2000. Rnx deficiency results in congenital central hypoventilation. Nat. Genet. 24: 287-290.

Suzuki, S.C., Inoue, T., Kimura, Y., Tanaka, T., and Takeichi, M. 1997. Neuronal circuits are subdivided by differential expression of type-II classic cadherins in postnatal mouse brains. Mol. Cell. Neurosci. 9: 433-447.

Tang, S.J., Hoodless, P.A., Lu, Z., Breitman, M.L., McInnes, R.R., Wrana, J.L., and Buchwald, M. 1998. The Tlx-2 homeobox gene is a downstream target of BMP signalling and is required for mouse mesoderm development. Development 125: $1877-1887$.

Tiveron, M.-C., Hirsch, M.-R., and Brunet, J.-F. 1996. The expression pattern of the transcription factor Phox2a delineates synaptic pathways of the autonomic nervous system. J. Neurosci. 16: 7649-7660.

Valarché, I., Tissier-Seta, J.-P., Hirsch, M.-R., Martinez, S., Goridis, C., and Brunet, J.-F. 1993. The mouse homeodomain protein Phox 2 regulates NCAM promoter activity in concert with Cux/CDP and is a putative determinant of neurotransmitter phenotype. Development 119: 881-896.

Yagi, T. and Takeichi, M. 2000. Cadherin superfamily genes: Functions, genomic organization, and neurologic diversity. Genes \& Dev. 14: 1169-1180.

Yokoyama, M., Nishi, Y., Yoshii, J., Okubo, K., and Matsubara, K. 1996. Identification and cloning of neuroblastoma-specific and nerve tissue-specific genes through compiled expression profiles. DNA Res. 3: 311-320. 


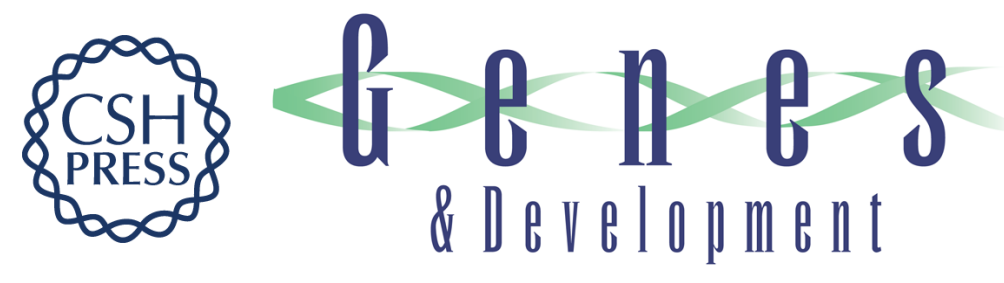

\section{Formation of brainstem (nor)adrenergic centers and first-order relay visceral sensory neurons is dependent on homeodomain protein Rnx/TIx3}

Ying Qian, Bernd Fritzsch, Senji Shirasawa, et al.

Genes Dev. 2001, 15:

Access the most recent version at doi:10.1101/gad.921501

$\begin{array}{ll}\text { References } & \begin{array}{l}\text { This article cites } 54 \text { articles, } 22 \text { of which can be accessed free at: } \\ \text { http://genesdev.cshlp.org/content/15/19/2533.full.html\#ref-list-1 }\end{array}\end{array}$

License

Email Alerting Receive free email alerts when new articles cite this article - sign up in the box at the top Service right corner of the article or click here.

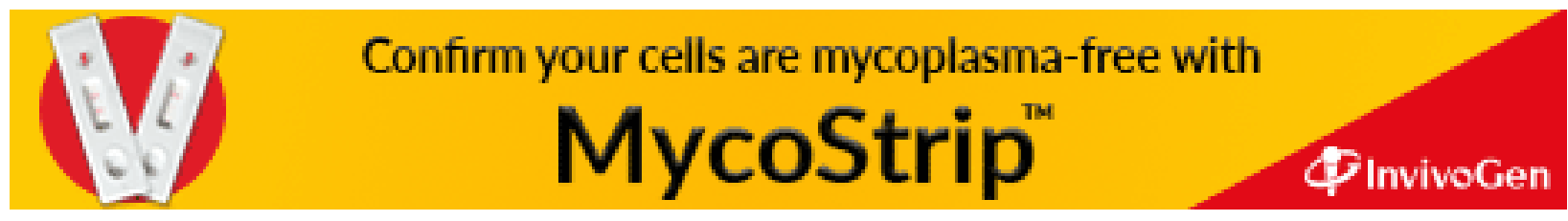

